TRANSACTIONS OF THE

AMERICAN MATHEMATICAL SOCIETY

Volume 361, Number 7, July 2009, Pages 3597-3610

S 0002-9947(09)04662-5

Article electronically published on March 3, 2009

\title{
GENERIC GROUPS ACTING ON REGULAR TREES
}

\author{
MIKLÓS ABÉRT AND YAIR GLASNER
}

\begin{abstract}
Let $T$ be a $k$-regular tree $(k \geq 3)$ and $A=\operatorname{Aut}(T)$ its automorphism group. We analyze a generic finitely generated subgroup $\Gamma$ of $A$. We show that $\Gamma$ is free and establish a trichotomy on the closure $\bar{\Gamma}$ of $\Gamma$ in $A$. It turns out that $\bar{\Gamma}$ is either discrete, compact or has index at most 2 in $A$.
\end{abstract}

\section{INTRODUCTION}

Let $T$ be a $k$-regular tree $(k \geq 3)$ and let $\operatorname{Aut}(T)$ be its automorphism group. The topology of pointwise convergence turns Aut $(T)$ into a locally compact, totally disconnected, unimodular topological group. Let $\mu$ be a Haar measure on Aut $(T)$ normalized so that vertex stabilizers have measure 1. Since $\operatorname{Aut}(T)$ is not compact, $\mu$ is an infinite measure. The group $\operatorname{Aut}(T)$ has a unique normal subgroup $\operatorname{Aut}^{0}(T)$ of index 2 , which is simple.

Let $n \geq 2$ be an integer. For the $n$-tuple $\mathbf{a}=\left(a_{1}, a_{2}, \ldots, a_{n}\right) \in \operatorname{Aut}(T)^{n}$ let $\langle\mathbf{a}\rangle=\left\langle a_{1}, a_{2}, \ldots, a_{n}\right\rangle$ denote the group generated by the $a_{i}$. We say that a group theoretic property $P$ is measure generic if $P$ holds for the group $\langle\mathbf{a}\rangle$ for $\mu^{n}$-almost all $\mathbf{a} \in \operatorname{Aut}(T)^{n}$. Similarly, $P$ is said to be topologically generic if it holds for the group $\langle\mathbf{a}\rangle$ for all but a meager (or first category) subset of $\operatorname{Aut}(T)^{n}$. The property $P$ is called generic if it is both measure and topologically generic. E.g. the sentence "A generic finitely generated subgroup of $\operatorname{Aut}(T)$ is infinite" means that for all $n \in \mathbb{N}$ the set $\left\{\mathbf{a} \in \operatorname{Aut}(T)^{n} \mid\langle\mathbf{a}\rangle\right.$ is finite $\}$ is a meager nullset.

The main result of this paper is the following.

Theorem 1. Let $n \geq 2$ and let $\Gamma<\operatorname{Aut}(T)$ be a generic subgroup on $n$ generators. Then $\Gamma$ is isomorphic to $F_{n}$, the free group of rank $n$ and it falls into exactly one of the following categories:

a) $\Gamma$ is discrete;

b) $\Gamma$ is precompact; that is, $\Gamma$ fixes a vertex or a geometric edge of $T$;

c) $\Gamma$ is dense in $\operatorname{Aut}(T)$ or in $\operatorname{Aut}^{0}(T)$.

We also show that all of a), b) and c) hold on a subset of infinite measure.

An essential tool in proving Theorem 1 is the natural action of the automorphism group $\operatorname{Aut}\left(F_{n}\right)$ on $\operatorname{Aut}(T)^{n}$ by measure-preserving homeomorphisms. We make use of a theorem of Weidmann implying that every nondiscrete finitely generated free subgroup of $\operatorname{Aut}(T)$ has a primitive element that fixes a vertex or a geometric edge.

Received by the editors March 8, 2007.

2000 Mathematics Subject Classification. Primary 20E08, 20E06; Secondary $20 B 15$.

Part of this work was carried out at the Institute for Advanced Studies and supported by NSF grant DMS-0111298. 
The group Aut $(T)$ and its discrete subgroups have been extensively studied in the literature (see [Tit70, [FTN91, Ser80], BaL00] and the references therein). Precompact subgroups, which are just groups acting on rooted trees, have also been thoroughly investigated (see Gri00, and for random generation Bha95, AV05. and references therein). The existence of finitely generated dense free subgroups in $\operatorname{Aut}(T)$ is a new phenomenon however, and it leads to a new example in the realm of infinite permutation groups.

Corollary 2. For all $n \geq 2$ the free group $F_{n}$ has a primitive, but not 2-transitive action on a countable set $X$, such that for each finite subset $Y \subseteq X$ the pointwise stabilizer $F_{Y}$ is nontrivial and every nontrivial subnormal subgroup of $F$ is transitive on $X$.

It is natural to ask whether the trichotomy established in Theorem 1 works for arbitrary locally compact groups. Namely, is it true that in such a group $G$ the closure of a generic subgroup is either open, compact or discrete. For connected semisimple Lie groups (such as $\mathrm{SL}(2, \mathbb{R})$ ) there is actually a dichotomy: generically, the closure is either open or discrete (see [Gel07] for a proof in the compact case). However, as we show in Proposition 17, the trichotomy is not true in general.

The paper is organized as follows. Section 2 introduces some notation and known results that will be needed later. In Section 3 we show that a fixed hyperbolic and a generic elliptic element generate a dense subgroup. This is the core result that, using Weidmann's theorem, leads to Theorem 1 that is proved in Section 4 . Finally, in Section 5 we apply our results; in particular, we obtain Corollary 2 and Proposition 17

\section{Tree AUTOMORPHisms}

Let $k \geq 3$ and let $T=T_{k}$ be a $k$-regular tree. For $x, y \in T$ let $[x, y]$ be the unique simple path going from $x$ to $y$ and let $d(x, y)$ be the length of $[x, y]$, the distance of $x$ and $y$ in the graph metric. If $x$ and $y$ are neighbours, let

$$
\text { Shadow }_{x \longrightarrow y}=\{t \in T \mid y \in[x, t]\}
$$

be the shadow of the directed edge $x \longrightarrow y$. For $t \in T$ and $n \in N$ let $B(t, n)=$ $\{x \in T \mid d(t, x) \leq n\}$.

Let $A=\operatorname{Aut}(T)$. Taking stabilizers of finite subsets of $T$ as a base of neighbourhoods of $1, A$ turns into a totally disconnected, locally compact, unimodular topological group. Thus $A$ admits a left and right invariant Haar measure $\mu$ and by normalizing we can assume that every vertex stabilizer has $\mu$-measure 1 .

The tree $T$ is a bipartite graph, and the stabilizer of the bipartite partition, $A_{0}=\operatorname{Aut}^{0}(T)$, is the unique nontrivial normal subgroup of $A$.

It is easy to see that a subgroup of $A$ is discrete if and only if it has finite vertex stabilizers. Also, a subgroup is precompact, i.e., it has a compact closure in $A$, if and only if it fixes a vertex or a geometric edge. By a geometric edge we mean a set of two adjacent vertices.

Let us briefly recall the classification of tree automorphisms. Elements of $A$ are either elliptic (fixing a vertex of $T$ ), inversions (fixing a geometric edge but not a vertex of $T$ ) or hyperbolic elements (the rest). For convenience, we want to treat inversions as elliptic elements. To do so, let us take the so-called barycentric subdivision of the tree $T$; that is, let us insert a new vertex in the center of each edge of $T$. The new tree obtained this way is bi-regular, and its automorphism 
group equals $A$. Indeed, the only thing that changes is that now inversions also fix a vertex, that is, they are elliptic. So, from now on, $T$ denotes this geometric realization and $A$ its automorphism group. The price we pay for this convenience is that we need to prove all our results in the realm of bi-regular trees.

For $a \in A$ let us define the minimal translation length as

$$
l(a)=\min _{x \in T}\{d(x, x a)\}
$$

and the support as

$$
X(a)=\{x \in T \mid d(x, x a)=l(a)\} .
$$

So, $a$ is elliptic if and only if $l(a)=0$. In this case $X(a)$ is a convex subtree of $T$ consisting of the fixed points of $a$. If $a$ is hyperbolic, then $X(a)$ is an $a$-invariant infinite geodesic called the axis of $a$ and $a$ acts on $X(a)$ by translation of length $l(a)$.

Now we will quote some classical results on groups acting on trees. The reader can find them e.g. in Serre's book on trees [Ser80].

The set of elliptic elements is clearly open, and therefore so is the set of hyperbolics. It is also well understood which subgroups consist of only elliptic or hyperbolic elements. A group consists of elliptic elements if and only if it fixes a vertex. This follows from the fact that if $a, b$ are elliptic with no common fixed point, then $a b$ is hyperbolic.

Let $X$ and $Y$ be convex subsets of $T$. Let us define the projection of $Y$ onto $X$ to be the set

$$
\operatorname{Proj}_{X} Y=\{x \in X \mid d(x, Y)=d(X, Y)\} .
$$

Let $H=\left\{h_{1}, h_{2}, \ldots, h_{n}\right\}$ be a set of hyperbolic elements and let $X_{i}=X\left(h_{i}\right)$. Then $H$ satisfies the $S$ chottky condition if for every $1 \leq i \leq n$ there exists a geodesic $Y_{i} \subset X_{i}$ of length at most $l\left(h_{i}\right)-1$ such that

$$
\bigcup_{j \neq i} \operatorname{Proj}_{X_{i}}\left(X_{j}\right) \subseteq Y_{i}
$$

Now a theorem of Lubotzky [Lub91] asserts that a finitely generated subgroup of $A$ is discrete and free if and only if it has a set of free generators satisfying the Schottky condition. This shows that a subgroup of $A$ consists only of hyperbolic elements if and only if it is discrete and free and that for a finite set of generators this is an open condition.

Now we will fix our convention to treat automorphisms of rooted trees. Let $d_{0}, d_{1}, \ldots, d_{n}$ be a sequence of positive integers ( $n$ might be infinite). Let $Z_{r}$ denote the set of residue classes modulo $r$. For $l<n+1$ let

$$
T_{l}=\left\{\left(s_{0}, s_{1}, \ldots, s_{l-1}\right) \mid s_{i} \in Z_{d_{i}}\right\} .
$$

The root is the empty sequence ()$\in T_{0}$. Let

$$
T=T\left(d_{0}, d_{1}, \ldots, d_{n}\right)=\bigcup_{0 \leq l<n+1} T_{l} .
$$

The level of a vertex $v \in T_{l}$ is defined to be $|v|=l$. A vertex $w=\left(r_{0}, r_{1}, \ldots, r_{m}\right) \in$ $T$ is a descendant of $v=\left(s_{0}, s_{1}, \ldots, s_{l}\right) \in T$ (or $w>v$ ) if $l<m$ and $r_{i}=s_{i}$ $(0 \leq i \leq l)$. The vertex $w$ is a child of $v$ if it is a descendant and $m=l+1$. Drawing edges along the child relation turns $T$ into a spherically homogeneus tree rooted at (). 
Let $v \in T$ be a vertex of level $l$. An elementary automorphism at $v$ is defined as a permutation $\alpha \in \operatorname{Sym}\left(Z_{d_{l}}\right)$, the symmetric group on $Z_{d_{l}}$ acting on $T$ as follows. For $w=\left(r_{0}, r_{1}, \ldots, r_{m}\right) \in T$ let

$$
w^{\alpha}=\left\{\begin{array}{cc}
\left(r_{0}, \ldots, r_{l-1}, r_{l}^{\alpha}, r_{l+1}, \ldots, r_{m}\right) & \text { if } w \geq v \\
w & \text { otherwise. }
\end{array}\right.
$$

Elementary automorphisms generate the full automorphism group $\operatorname{Aut}(T)$ (if $n$ is infinite, then one has to consider topological generation). Furthermore, every automorphism $\varphi \in \operatorname{Aut}(T)$ can be uniquely written as a product of elementary automorphisms

$$
\varphi=\prod_{v \in T} \varphi(v),
$$

where $\varphi(v)$ is an elementary automorphism at $v$ and the product is taken in nondecreasing order with respect to the level of $v$. If $n$ is infinite, then the above infinite product should be understood as a limit.

Note that elementary automorphisms at incomparable vertices commute, so we can rearrange our product accordingly. We will choose a rearrangement that reflects looking at $\operatorname{Aut}(T)$ as the wreath product of $\operatorname{Aut}\left(T\left(d_{l}, d_{l+1}, \ldots, d_{n}\right)\right)$ with $\operatorname{Aut}\left(T\left(d_{0}, d_{1}, \ldots, d_{l-1}\right)\right)$ as follows. For $1 \leq l<n$ and $\varphi \in \operatorname{Aut}(T)$ let

$$
\varphi_{l}=\prod_{|v|<l} \varphi(v)
$$

and for $w \in T$ of level $l$ let

$$
\varphi_{w}=\prod_{v \geq w} \varphi(v),
$$

again in nondecreasing order with respect to the level of $v$. Then

$$
\varphi=\varphi_{l} \prod_{|v|=l} \varphi_{v}
$$

where the product is now in arbitrary order since the different $\varphi_{v}$-s commute. The autmorphism $\varphi_{l}$ acts on the union of the first $l$ levels of $T$, so it can be looked at as an element of $\operatorname{Aut}\left(T\left(d_{0}, d_{1}, \ldots, d_{l-1}\right)\right)$. The automorphism $\varphi_{v}$ acts on the subtree $T\left(d_{l}, d_{l+1}, \ldots, d_{n}\right)$; this action is called the state of $\varphi$ at $v$ and is also denoted by $\varphi_{v}$.

Let $G$ be any group and let $n$ be an integer. Then $\operatorname{Aut}\left(F_{n}\right)$ has a natural action on $\operatorname{Hom}\left(F_{n}, G\right)$ as follows. For $\varphi \in \operatorname{Aut}\left(F_{n}\right)$ and $f \in \operatorname{Hom}\left(F_{n}, G\right)$ let

$$
f^{\varphi}: w \longmapsto f\left(w^{\varphi}\right)\left(w \in F_{n}\right) .
$$

By fixing a minimal generating set for $F_{n}$ we can look at $\operatorname{Hom}\left(F_{n}, G\right)$ as the set of $n$-tuples from $G$. Hence Aut $\left(F_{n}\right)$ acts on $G^{n}$ as well. This action can be best understood by Nielsen transformations, the action of the Nielsen generators of $\operatorname{Aut}\left(F_{n}\right)$. Let $\mathbf{a}=\left(a_{1}, a_{2}, \ldots, a_{n}\right) \in G^{n}$. Then a right Nielsen transformation of $G^{n}$ is of the form

$$
\left(a_{1}, \ldots, a_{n}\right)^{R_{i, j}^{ \pm}}=\left(a_{1}, \ldots, a_{i-1}, a_{i} a_{j}^{ \pm 1}, a_{i+1}, \ldots, a_{n}\right)(i \neq j),
$$

and a left Nielsen transformation of $G^{n}$ is

$$
\left(a_{1}, \ldots, a_{n}\right)^{L_{i, j}^{ \pm}}=\left(a_{1}, \ldots, a_{i-1}, a_{j}^{ \pm 1} a_{i}, a_{i+1}, \ldots, a_{n}\right)(i \neq j) .
$$


Lastly, replacing any one of the coordinates by its inverse is also considered a Nielsen transformation.

It turns out that if the generators a are chosen uniform randomly, then the image will still be distributed in the same way. This is true in both the measure theoretic and the topological settings by the following.

Lemma 3. Let $G$ be a unimodular locally compact topological group with a Haar measure $\mu$. Then Nielsen transformations act on $G^{n}$ by $\mu$-preserving homeomorphisms.

Proof. For permutations of coordinates, the lemma is trivial. Clearly $R_{i, j}^{ \pm}$and $L_{i, j}^{ \pm}$ are continuous bijections of $G^{n}$. Since their inverses are also Nielsen transformations, they are homeomorphisms of $G^{n}$. To demonstrate that Nielsen transformations are measure preserving, it is enough to check the 2 variable case $N:(x, y) \longmapsto$ $(x y, y)$. Using Fubini's theorem, we have

$$
\begin{aligned}
\int_{G \times G} f \circ N(x, y) d \mu^{2}(x, y) & =\int_{G} \int_{G} f(x y, y) d \mu(x) d \mu(y) \\
& =\int_{G} \int_{G} f(x, y) d \mu(x) d \mu(y) \\
& =\int_{G \times G} f(x, y) d \mu^{2}(x, y) .
\end{aligned}
$$

Note that we only used the right invariance of $\mu$ for this calculation. For left Nielsen transformations we have to use the left invariance of $\mu$.

We say that two $n$-tuples $a, b \in G^{n}$ are Nielsen-equivalent if there is a sequence of Nielsen transformations leading from $a$ to $b$, or, equivalently, if they lie in the same $\operatorname{Aut}\left(F_{n}\right)$-orbit.

A crucial fact about Nielsen transformations is that they do not change the subgroup that the $n$-tuple generates. On the other hand, they do change the tuple considerably, allowing us to find the 'right' generating set for the subgroup. This method is called the Nielsen method; it was first used by Nielsen to show that finitely generated subgroups of a free group are free Nie55. Since then, the method has been further developed by Zieschang, Weidmann, Kapovich and others. We will need the following theorem that can be found in Weidmann's paper Wei02, Theorem 7], setting $S_{i}=\emptyset$.

Theorem 4 (Weidmann). Let $\Gamma=\left\langle\gamma_{1}, \gamma_{2}, \ldots, \gamma_{n}\right\rangle$ be a finitely generated group acting on a tree. Then either $\Gamma$ is a free group acting freely on $T$ or there is a Nielsen equivalent set of generators $\Gamma=\left\langle\gamma_{1}^{\prime}, \gamma_{2}^{\prime}, \ldots, \gamma_{n}^{\prime}\right\rangle$ such that $\gamma_{1}^{\prime}$ is elliptic.

\section{The hyperbolic-ELLIPTIC CASE}

In this section we show that a group generated by an elliptic and a hyperbolic element is generically dense. In fact, we derive this from the stronger result, that the group $\Gamma$ generated by a fixed hyperbolic element and a generic elliptic element is dense. The essence of the proof is to show that vertex stabilizers of $\Gamma$ are dense in the automorphism group of the tree rooted at the vertex. This proceeds by using conjugates of high powers of the elliptic element by powers of the hyperbolic element. Although these rooted automorphisms will not be independent random, we shall be able to pull up sufficient independence to ensure denseness. 
Let us start with some notations and basic results on random actions on rooted trees.

Let $U$ be a spherically homogeneous locally finite infinite rooted tree. Then $U$ is isomorphic to $T\left(d_{0}, d_{1}, \ldots\right)$, where $d_{i}$ is the number of children of a vertex at level $i$. For $1 \leq l$ let

$$
U_{l}=T\left(d_{l}, d_{l+1}, \ldots\right)
$$

denote the subtree of $U$ hanging down from a vertex of level $l$ and let

$$
U^{l}=T\left(d_{0}, d_{1}, \ldots, d_{l-1}\right)
$$

be the union of the first $l$ levels of $U$.

Following the elementary decomposition (1), a Haar uniform random element $a \in \operatorname{Aut}(U)$ will decompose as

$$
a=\prod_{v \in T} a(v)
$$

where $a(v)$ is a uniform random element of the finite group $\operatorname{Sym}\left(Z_{a_{l}}\right)$ with $l=|v|$. This implies that for all $l \geq 1$ the component $a_{l}$ is a uniform random element of $\operatorname{Aut}\left(U^{l}\right)$ and for all vertices $v \in V$ the component $a_{v}$ is a uniform random element of $\operatorname{Aut}\left(U_{l}\right)$. Also, the set

$$
\left\{a_{u} \mid u \in U \text { of level } l\right\}
$$

will consist of independent uniform random elements of $\operatorname{Aut}\left(U_{l}\right)$.

For an element $a \in \operatorname{Aut}(U)$ and a vertex $u \in U$ let $a \circ u$ denote the action of $a^{m}$ on $U_{l}$ where $m$ is the minimal positive integer such that $a^{m}$ fixes $u$.

Our first lemma already appears in a slightly weaker form in AV05.

Lemma 5. Let a be a uniform random element of $\operatorname{Aut}(U)$. Then for each $u \in U$, $a \circ u$ is a uniform random element of $\operatorname{Aut}\left(U_{l}\right)$ where $l=|u|$.

Proof. Let $u_{0}=u$ and $u_{i}=u_{0}^{a^{i}}(1 \leq i<m)$. Let us fix this configuration. Then the $a_{u_{i}}(0 \leq i<m)$ are independent random elements of $\operatorname{Aut}\left(U_{l}\right)$. Now

$$
\begin{aligned}
a^{m} & =\left(a_{l} \prod_{|v|=l} a_{v}\right)^{m} \\
& =a_{l}^{m}\left(a_{l}^{-(m-1)}\left(\prod_{|v|=l} a_{v}\right) a_{l}^{m-1}\right) \cdots\left(a_{l}^{-1}\left(\prod_{|v|=l} a_{v}\right) a_{l}\right) \prod_{|v|=l} a_{v} .
\end{aligned}
$$

We know that $a_{l}^{m}$ fixes $u$ and

$$
a_{l}^{-i}\left(\prod_{|v|=l} a_{v}\right) a_{l}^{i}=\prod_{|v|=l} b_{v}, \text { where } b_{v}=a_{w} \text { with } w=v^{a_{l}^{i}}
$$

This implies that

$$
a \circ u=\left(a^{m}\right)_{u}=\prod_{i=m-1}^{0} a_{u_{i}}
$$

the product of independent uniform random elements of $\operatorname{Aut}\left(U_{l}\right)$, which is then also uniform random. 
Note that if $u$ and $v$ lie in the same $a$-orbit, then $a \circ u$ and $a \circ v$ are actually conjugate (being the same product up to a cyclic permutation), so they are very far from being independent.

We will need to mine out much more independence. To achieve this, we will take an increasing sequence of vertices $u_{i}$ and look at the action of $a \circ u_{i}$ at the first $n$ levels of the tree $U_{l}$.

Lemma 6. Let a be a uniform random element of $\operatorname{Aut}(U)$. Let $u_{1}<u_{2}<\ldots$ be an infinite descending sequence of vertices such that $\left|u_{i+1}\right|-\left|u_{i}\right| \geq L(i \geq 1)$. Assume that the trees $\left(U_{u_{i}}\right)^{L}$ of length $L$ are all isomorphic to

$$
V=T\left(e_{0}, e_{1}, \ldots, e_{L-1}\right) .
$$

Then the set

$$
\left\{\left(a \circ u_{i}\right)_{L} \mid i \geq 1\right\}
$$

consists of independent uniform random elements of $\operatorname{Aut}(V)$.

Proof. Let $k_{i}=\left|u_{i}\right|(i \geq 1)$. We have seen in Lemma 5 that $a \circ u_{i}$ is a uniform random element of $\operatorname{Aut}\left(U_{u_{i}}\right)(i \geq 1)$. This implies that $\left(a \circ u_{i}\right)_{L} \in \operatorname{Aut}(V)$ will also be uniform random. Note that $\left(a \circ u_{i}\right)_{L}$ only depends on the values $a(v)$ where $|v|<k_{i}+L$, since the elementary automorphisms at higher level vertices fix the relevant tree $U^{k_{i}+L-1}$.

Let $r \geq 1$. Fix the value of $a_{k_{r}}$. As shown above, this will determine the value of $\left(a \circ u_{i}\right)_{L}(i<r)$. The proof of Lemma 5 shows that $\left(a \circ u_{r}\right)_{L}$ is a function of the set

$$
S=\left\{a(v)\left|k_{r} \leq\right| v \mid<k_{r}+L\right\} .
$$

Note that the actual function is governed by the orbit structure of $a_{k_{r}}$, so it does depend on the values of $a(v)\left(|v|<k_{r}\right)$, but since those are fixed, it is a fixed function of the random variables in $S$. Now Lemma 5 tells us that $\left(a \circ u_{r}\right)_{L} \in \operatorname{Aut}(V)$ is uniform random.

We obtained the fact that for any fixed value of

$$
\left(a \circ u_{i}\right)_{L}(i<r)
$$

the distribution of $\left(a \circ u_{r}\right)_{L}$ is uniform random. Using induction on $r$, this implies that the set

$$
\left\{\left(a \circ u_{i}\right)_{L} \mid 1 \leq i \leq r\right\}
$$

consists of independent uniform random elements of $\operatorname{Aut}(V)$.

We need the following technical lemma on rooted trees.

Lemma 7. Let $T=T\left(e_{0}, e_{1}, \ldots, e_{L-1}\right)$ be a rooted tree with $e_{0} \geq 3$. Let $x$ and $y$ be distinct vertices of $T$ on the first level. Let

$$
X=\{g \in \operatorname{Aut}(V) \mid z g=z \forall z \geq x\}
$$

and

$$
Y=\{g \in \operatorname{Aut}(V) \mid z g=z \forall z \geq y\} .
$$

Then $X$ and $Y$ generate $\operatorname{Aut}(V)$.

Proof. Let $v \in T$ be a vertex distinct from the root. Then $v \nsupseteq x$ or $v \nsupseteq y$, so all elementary automorphisms at $v$ are contained in $X \cup Y$. Since $e_{0} \geq 3$, the 


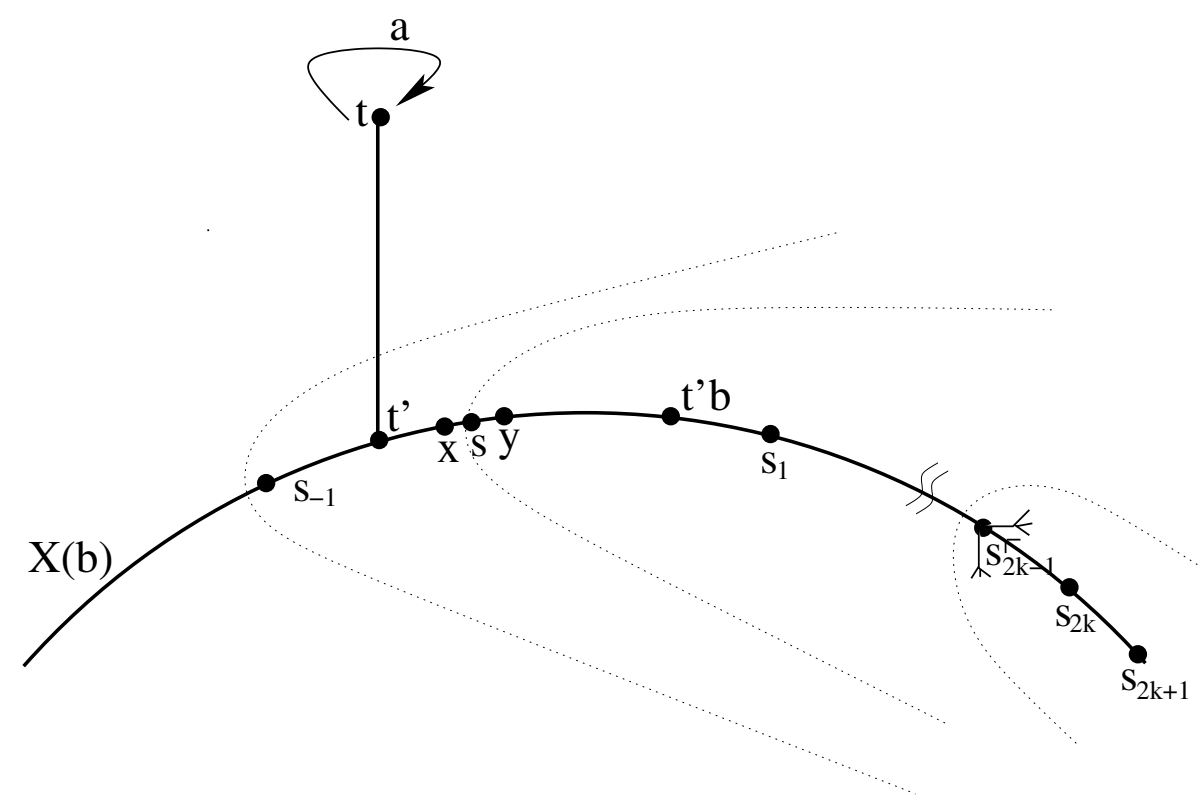

FiguRe 1. Mining out randomness

symmetric group $\operatorname{Sym}\left(e_{0}\right)$ is generated by any two distinct point stabilizers. This implies that any elementary automorphism at the root is generated by elements of $X$ and $Y$. Thus all elementary automorphisms are generated by $X \cup Y$, and the lemma holds.

Now we start to discuss random generation for unrooted trees. For a vertex $t \in T$ let $\operatorname{Aut}(T)_{t}$ denote the stabilizer of $t$ in $\operatorname{Aut}(T)$.

Lemma 8. Let $b \in \operatorname{Aut}(T)$ be hyperbolic and let $t \in T$ be a vertex. Let $a \in \operatorname{Aut}(T)_{t}$ be a random element and let

$$
\Gamma=\langle a, b\rangle \leq \operatorname{Aut}(T) .
$$

Then generically, there exists $s \in X(b)$ of degree at least 3 such that the stabilizer $\Gamma_{s}$ is dense in $\operatorname{Aut}(T)_{s}$.

Proof. Let $l=l(b)$ be the translation distance of $b$. Let $t^{\prime}$ be the projection of $t$ on $X(b)$ and let $s$ be an element of the geodesic $\left(t^{\prime}, t^{\prime} b\right]$ with degree at least 3 . Let $x \in\left[t^{\prime}, s\right]$ with $d(x, s)=1$ and let $y \in[s, s b]$ with $d(s, y)=1$. Let $T_{s}$ be the tree $T$ rooted at $s$.

Let us fix a positive integer $K$. We claim that generically, the action of $\Gamma_{s}$ on $T_{s}^{K}$ equals the full automorphism group $\operatorname{Aut}\left(T_{s}^{K}\right)$. For an integer $n$ let

$$
s_{n}=s b^{K n}, \text { let } x_{n}=x b^{K n}, \text { let } y_{n}=y b^{K n}
$$

and let

$$
V_{i}=\text { Shadow }_{x_{2 i-1} \longrightarrow s_{2 i-1}} \text { and } W_{i}=\text { Shadow }_{y_{2 i-1} \longrightarrow s_{2 i-1}}
$$

as trees rooted at $s_{2 i-1}$. 


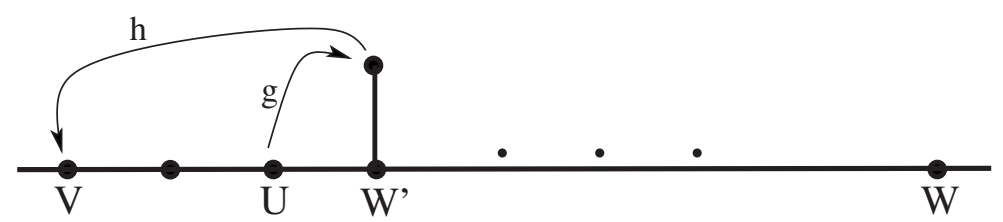

FiguRE 2. Transitive action on the even vertices

Let us apply Lemma 6 for the tree $U=T$ (rooted at $t$ ), setting $L=2 K$ and $u_{i}=s_{2 i-1}(i \geq 1)$. We get that the set

$$
\left\{\left(a \circ u_{i}\right)_{L} \mid i \geq 1\right\}
$$

consists of independent uniform random elements of $\operatorname{Aut}\left(V_{i}^{L}\right)$.

Let $g$ be an arbitrary element of $\operatorname{Aut}\left(V_{-1}^{L}\right)$ that fixes every vertex of $V_{-1}^{L}$ that is not a descendant of $s$. Then because of the above independence, generically there exists $i_{g} \geq 1$ such that

$$
a_{g}=b^{2 K i_{g}}\left(a \circ u_{i_{g}}\right) b^{-2 K i_{g}} \in \Gamma_{s}
$$

acts on $V_{-1}^{L}$ as $g$ does. Let $z \in T_{s}^{K}$ with $z \geq x$. Then $d(z, s) \leq K$ and $d\left(s_{-1}, s\right)=K$, so $d\left(s_{-1}, z\right) \leq 2 K$, which implies $z a_{g}=z$.

The same way, applying Lemma 6 for $u_{i}=s_{-2 i+1}(i \geq 1)$ we get that for an arbitrary element $h \in \operatorname{Aut}\left(W_{1}^{L}\right)$ that fixes every vertex of $W_{1}^{L}$ that is not a descendant of $s$, generically there exists $i_{h} \geq 1$ such that

$$
a_{h}=b^{-2 K i_{h}}\left(a \circ u_{i_{h}}\right) b^{2 K i_{h}} \in \Gamma_{s}
$$

acts on $W_{1}^{L}$ as $h$ does. Also, for all $z \in T_{s}^{K}$ with $z \geq y$ we have $z a_{h}=z$.

Applying Lemma 7 on the tree $T_{s}^{K}$ we get that the actions of all the $a_{g}$ and $a_{h}$ on $T_{s}^{K}$ generate $\operatorname{Aut}\left(T_{s}^{K}\right)$, and so our claim holds.

Since for all $K$ the claim generically holds, it generically holds for all $K$ at the same time. In particular, $\Gamma_{s}$ is generically dense in $\operatorname{Aut}(T)_{s}$.

The following lemma will be used to establish densenesss in $\operatorname{Aut}^{0}(T)$. To make the notation simpler, we will use regular trees here (that is, we forget the baricentric points).

Lemma 9. Let $\Gamma$ be a subgroup of $\operatorname{Aut}(T)$. Assume that $\Gamma$ contains a hyperbolic element and that there exists $s \in T$ such that $\Gamma_{s}$ is dense in $\operatorname{Aut}(T)_{s}$. Then the closure of $\Gamma$ contains $\operatorname{Aut}^{0}(T)$.

Proof. Let $b$ be a hyperbolic element of $\Gamma$. Let $X$ be the set of vertices that have even distance from $s$. Let $Y$ be the $\Gamma$-orbit of $s$. Using conjugation, it is easy to see that for all $y \in Y$, the stabilizer $\Gamma_{y}$ is dense in $\operatorname{Aut}(T)_{y}$.

We claim that $X \subseteq Y$. To show this, let $u \in X \cap Y$ and let $v \in T$ such that $d(u, v)=2$. Let $w=s$ or $w=s b$ such that $w \neq u$. Let $w^{\prime} \in[u, w]$ adjacent to $u$ and let $v^{\prime} \notin[u, w]$ be a vertex that is adjacent to $w^{\prime}$. Such $v^{\prime}$ exists since the degree of $w^{\prime}$ is at least 3. Now using density at $w$ and $d(w, u)=d\left(w, v^{\prime}\right)$, there exists an element $g \in \Gamma_{w}$ with $u g \in v^{\prime}$. This implies $v^{\prime} \in X \cap Y$. Now $d(u, v)=d\left(u, v^{\prime}\right)=2$, so using density again at $u$, there exists $h \in \Gamma_{u}$ with $v^{\prime} h \in v$, implying $v \in X \cap Y$. Thus every element of distance 2 from an element in $X \cap Y$ lies in $Y$ and the claim holds. 
Let $G$ be the closure of $\Gamma$ in $\operatorname{Aut}(T)$. The group $\operatorname{Aut}^{0}(T)$ acts transitively on $X$, and for all $x \in X$ the stabilizers

$$
G_{x}=\operatorname{Aut}^{0}(T)_{x}=\operatorname{Aut}(T)_{x} .
$$

So if $X=Y$, then $G=\operatorname{Aut}^{0}(T)$; otherwise $G=\operatorname{Aut}(T)$.

We are ready to establish the main result of this section. Note that we do use the baricentric subdivision here; that is, we allow vertices of degree 2 .

Theorem 10. Let a be a generic elliptic and let $b$ be a generic hyperbolic element of $\operatorname{Aut}(T)$. Then

$$
\operatorname{Aut}^{0}(T) \subseteq \overline{\langle a, b\rangle} .
$$

Proof. Let $b$ be a fixed hyperbolic element of $\operatorname{Aut}(T)$, let $t \in T$ and let $a$ be a generic element of $\operatorname{Aut}(T)_{t}$. Let $\Gamma=\langle a, b\rangle$ and let $G$ be the closure of $\Gamma$ in $\operatorname{Aut}(T)$. Using Lemma 8 there exists $s \in X(b)$ of degree at least 3 such that the stabilizer $\Gamma_{s}$ is dense in $\operatorname{Aut}(T)_{s}$. Since $s$ is not a baricenter, we can use Lemma 9, which implies $\operatorname{Aut}^{0}(T) \subseteq G$.

The measure theoretic part of the theorem now follows by summing over $t$ and integrating over the hyperbolic coordinate. Let Ell denote the set of elliptic and Hyp the set of hyperbolic elements in Aut $(T)$. By Baire's theorem, to establish the topological statement, it is enough to show that for any $\phi \in \operatorname{Aut}^{0}(T)$ and any $n \in N$ the set

$$
W(n, \phi)=\left\{(a, b) \in \operatorname{Hyp} \times \operatorname{Ell} \mid \exists \psi \in\langle a, b\rangle \text { such that } \phi\left|B\left(t_{0}, n\right)=\psi\right| B\left(t_{0}, n\right)\right\}
$$

is a dense open subset of the set Hyp $\times$ Ell. Recall that $B(t, n)$ denotes the ball of radius $n$ around $t$. The set $W(n, \phi)$ is open, because if the word $\psi=w(a, b)$ realizes the condition $\phi\left|B\left(t_{0}, n\right)=\psi\right| B\left(t_{0}, n\right)$, then so will $\psi^{\prime}=w\left(a^{\prime}, b^{\prime}\right)$ if $a^{\prime}$ and $b^{\prime}$ are sufficiently close to $a$ and $b$, respectively. The density of $W(n, \phi)$ follows from the measure theoretic part.

The theorem holds.

\section{TRIсHотому}

In this section we establish Theorem 1 First we need some notation.

Let

$$
\begin{aligned}
\operatorname{Ell}_{n} & =\left\{\mathbf{a} \in A^{n} \mid \mathbf{a}_{i} \text { is elliptic }(1 \leq i \leq n)\right\}, \\
\operatorname{Hyp}_{n} & =\left\{\mathbf{a} \in A^{n} \mid \mathbf{a}_{i} \text { is hyperbolic }(1 \leq i \leq n)\right\}, \\
\operatorname{Mix}_{n} & =A^{n} \backslash\left(\operatorname{Ell}_{n} \cup \operatorname{Hyp}_{n}\right) .
\end{aligned}
$$

Also let

$$
\begin{aligned}
C & =\left\{\mathbf{a} \in \operatorname{Ell}_{n} \mid\langle\mathbf{a}\rangle \text { is precompact }\right\}, \\
S & =\left\{\mathbf{a} \in \operatorname{Hyp}_{n} \mid \mathbf{a} \text { satisfies the Schottky-condition }\right\}, \\
D & =\left\{\mathbf{a} \in \operatorname{Mix}_{n} \mid\langle\mathbf{a}\rangle \text { is dense in } A \text { or } A^{0}\right\} .
\end{aligned}
$$

It is easy to see that $C, S$ and $D$ are pairwise disjoint, $C$ is open and closed, and $S$ is open.

Lemma 11. $D$ is generic and co-meager in $\operatorname{Mix}_{n}$; that is, $\operatorname{Mix}_{n} \backslash D$ is meager of measure 0 . 
Proof. For $n=2$ this is Theorem 10. For $n>2$ and $1 \leq i, j \leq n$ let

$$
E_{i j}=\left\{\mathbf{a} \in \operatorname{Mix}_{n} \backslash D \mid \mathbf{a}_{i} \text { is hyperbolic and } \mathbf{a}_{j} \text { is elliptic }\right\} .
$$

Using Theorem 10 the projection of $E_{i j}$ to the coordinates $i, j$ is meager of measure zero in $A^{2}$. So $E_{i j}$ is also meager of measure zero. The lemma now follows from

$$
\operatorname{Mix}_{n} \backslash D=\bigcup_{1 \leq i, j \leq n} E_{i j} .
$$

Let $\Delta$ denote the Nielsen action of $\operatorname{Aut}\left(F_{n}\right)$ on $A^{n}$. Since the action does not change the subgroup generated by the tuple, $C$ and $D$ are invariant under $\Delta$.

Lemma 12. We have $S^{\Delta} \subseteq \operatorname{Hyp}_{n}$.

Proof. If a satisfies the Schottky-condition, then $\langle\mathbf{a}\rangle$ is discrete and free. That is, vertex stabilizers are finite, which, using the fact that the free group is torsion-free, implies that they are trivial. So $\langle\mathbf{a}\rangle$ contains no elliptic elements. The same holds for $\left\langle\mathbf{a}^{\varphi}\right\rangle=\langle\mathbf{a}\rangle$, in particular, all the entries of $\mathbf{a}^{\varphi}$ are hyperbolic.

Lemma 13. We have

$$
S^{\Delta}=\left\{\mathbf{a} \in A^{n} \mid\langle\mathbf{a}\rangle \text { is discrete and free }\right\} .
$$

Proof. This follows from Lubotzky's theorem on the Schottky condition (see Section 2 ) and the fact that any two minimal generating sets of a free group are Nielsenequivalent.

Lemma 14. For $\mathbf{a} \in \operatorname{Ell}_{n} \backslash C$ there exists a Nielsen transformation $\varphi \in \Delta$ such that $\mathbf{a}^{\varphi} \in \operatorname{Mix}_{n}$.

Proof. Since $\mathbf{a} \notin C$, there are no common fixed points for the $\mathbf{a}_{i}$. Since the fixedpoint sets of the $\mathbf{a}_{i}$ are convex, it follows from the Caratheodory theorem on trees that there is $i, j$ such that $\mathbf{a}_{i}$ and $\mathbf{a}_{j}$ have no common fixed points. But this yields that $\mathbf{a}_{i} \mathbf{a}_{j}$ is hyperbolic, implying $\mathbf{a}^{R_{i, j}^{+}} \in \operatorname{Mix}_{n}$

Lemma 15. For $\mathbf{a} \in \operatorname{Hyp}_{n} \backslash S^{\Delta}$ there exists $\varphi \in \Delta$ such that $\mathbf{a}^{\varphi} \in \operatorname{Mix}_{n}$.

Proof. Lemma 13 implies that $\langle\mathbf{a}\rangle$ is not discrete and free. Then the theorem of Weidmann (Theorem (4) tells us that there is $\xi \in \Delta$ such that the tuple $\mathbf{a}^{\xi}$ contains an elliptic element. If $\mathbf{a}^{\xi} \in \operatorname{Mix}_{n}$, we proved our lemma. If $\mathbf{a}^{\xi} \in \mathrm{Ell}_{n}$, then $\mathbf{a}^{\xi} \notin C$; otherwise $\left\langle\mathbf{a}^{\xi}\right\rangle$ would consist of elliptic elements, contradicting $\mathbf{a} \in \operatorname{Hyp}_{n}$. So $\mathbf{a}^{\xi} \in \operatorname{Ell}_{n} \backslash C$, and using Lemma 14 we see that there exists $\delta \in \Delta$ such that $\mathbf{a}^{\xi \delta} \in \operatorname{Mix}_{n}$.

We are ready to prove Theorem 1.

Proof of Theorem 1. Let $\mathbf{a} \in A^{n}$ be a generic $n$-tuple. First we show that a generates a free group of rank $n$. The measure generic part directly follows from a result of the first author Ab05. Corollary 1.6]. For the topological part, let $w$ be a nontrivial word in $n$ letters. Then the support

$$
\operatorname{Supp}(w)=\left\{\mathbf{a} \in A^{n} \mid w(a)=1\right\}
$$

is closed in $A^{n}$, and by the measure theoretic part it has measure zero. This implies that it is nowhere dense. Hence the set of points satisfying any nontrivial words is meager, and so the topological part follows. 
Now we establish the trichotomy. Let

$$
L=\left(\operatorname{Mix}_{n} \backslash D\right)^{\Delta} .
$$

Then using Lemma 11, $L$ is a union of countably many $\operatorname{Mix}_{n} \backslash D$-translates, so $\mu(L)=0$ and $L$ is meager.

Let $\mathbf{a} \notin L$. We claim that there exists $\varphi \in \Delta$ such that $\mathbf{a}^{\varphi} \in C \cup D \cup S$.

If $\mathbf{a} \in \operatorname{Ell}_{n}$ and $\mathbf{a} \in C$, we are done. If $\mathbf{a} \in \operatorname{Ell}_{n} \backslash C$, then by Lemma 14there exists a Nielsen transformation $\varphi \in \Delta$ such that $\mathbf{a}^{\varphi} \in \operatorname{Mix}_{n}$. However, $\mathbf{a}^{\varphi} \notin L \supseteq \operatorname{Mix}_{n} \backslash D$, implying $\mathbf{a}^{\varphi} \in D$.

If $\mathbf{a} \in \operatorname{Hyp}_{n}$ and $\mathbf{a} \in S^{\Delta}$, then there exists a $\varphi \in \Delta$ such that $\mathbf{a}^{\varphi} \in S$. Otherwise $\mathbf{a} \in \operatorname{Hyp}_{n} \backslash S^{\Delta}$, and by Lemma 15 there exists $\varphi \in \Delta$ such that $\mathbf{a}^{\varphi} \in \operatorname{Mix}_{n}$. Again, $\mathbf{a}^{\varphi} \notin L \supseteq \operatorname{Mix}_{n} \backslash D$, so $\mathbf{a}^{\varphi} \in D$.

Finally, if $\mathbf{a} \in \operatorname{Mix}_{n}$, then $\mathbf{a} \notin L \supseteq \operatorname{Mix}_{n} \backslash D$ so $\mathbf{a} \in D$. We have proved our claim.

Since the group generated by $\mathbf{a}^{\varphi}$ equals the group generated by $\mathbf{a}$, the trichotomy holds.

Remark. The freeness of a generic subgroup implies that the action of $\operatorname{Aut}\left(F_{n}\right)$ on $\operatorname{Aut}(T)^{n}$ is essentially free. Another consequence is that apart from a nullset in $\operatorname{Aut}(T)^{n}$, the $\operatorname{Aut}\left(F_{n}\right)$-orbits can be identified with the subgroup that an element of the orbit generates. This allows us to talk about generic subgroups rather than the group generated by a generic $n$-tuple.

\section{Applications}

In this section we use the existence of dense free subgroups in $\operatorname{Aut}^{0}(T)$ to find some interesting permutation actions on countable sets. Then we discuss why the trichotomy theorem does not hold in general in the realm of locally finite groups.

Let us recall some notions of group actions. Let the group $\Gamma$ act on the set $\Omega$. The action is $k$-transitive if the action of $\Gamma$ on the ordered $k$-tuples of distinct elements of $\Omega$ is transitive. The action is primitive if there is no nontrivial $\Gamma$ invariant equivalence relation on $\Omega$. This is equivalent to saying that the action is transitive and a point stabilizer $\Gamma_{\omega}$ is a maximal subgroup in $\Gamma$. The action of $\Gamma$ is quasi-primitive if every normal subgroup of $\Gamma$ acts transitively or trivially. It is easy to see that every 2-transitive action is primitive and every primitive action is quasiprimitive. Primitive actions of finite and infinite groups have a well-established theory. Quasi-primitive actions of finite groups have also been extensively studied (see $\mathrm{Pra03}$ and references therein).

Let $G$ be a group. A subgroup $H \leq G$ is subnormal if there is a chain

$$
H=G_{n} \triangleleft G_{n-1} \triangleleft \ldots \triangleleft G_{0}=G .
$$

We say that the action of $\Gamma$ is subnormal transitive if every nontrivial subnormal subgroup of $\Gamma$ acts transitively or trivially. It is easy to see that primitive actions are not necessarily subnormally transitive, but if we assume that the group is $k$ transitive for every $k$, then it is also subnormal transitive.

The following general lemma will establish subnormal transitivity for a general class of actions.

Lemma 16. Let $G$ be a disconnected topologically simple topological group and let $O<G$ be an open subgroup. Let $\Gamma<G$ be a dense subgroup and let $\Lambda=\Gamma \cap O$. Then the right coset action of $\Gamma$ on $\Gamma / \Lambda$ is faithful and subnormal transitive. 
Proof. Let $N \neq 1$ be a subnormal subgroup of $\Gamma$. Then the closure of $N$ in $G$ is subnormal in the closure of $\Gamma$ in $G$, which is equal to $G$, since $\Gamma$ is dense in $G$. But $G$ is topologically simple, implying that $N$ is dense in $G$. Now using the fact that $O$ is open, we get $N O=G$. This implies

$$
N \Lambda=N(\Gamma \cap O)=\Gamma
$$

which is equivalent to saying that $N$ acts transitively on $\Gamma / \Lambda$.

Proof of Corollary 2, Let $T$ be the 3-regular infinite tree. Let $\Gamma$ be a generic $n$ generated subgroup of $\operatorname{Aut}(T)$. Then using Theorem 1, $\Gamma$ is free on $n$ generators, and on a set of infinite measure its closure in $\operatorname{Aut}(T)$ equals $\operatorname{Aut}^{0}(T)$. In particular, there exists a subgroup $\Gamma \subseteq \operatorname{Aut}^{0}(T)$ which is isomorphic to $F_{n}$ and dense in $\operatorname{Aut}^{0}(T)$.

Let $t \in T$ be a vertex, let $G=\operatorname{Aut}^{0}(T)$ and let $O=\operatorname{Aut}^{0}(T)_{t}$. Applying Lemma 16 we get that the right coset action of $\Gamma$ on $\Gamma / \Lambda$ is faithful and subnormal transitive, where $\Lambda=\Gamma_{t}$ is the vertex stabilizer of $\Gamma$. This right coset action is permutation isomorphic to the action of $\Gamma$ on $T$ and $\Gamma$ is dense in $\operatorname{Aut}^{0}(T)$, so the action of $\Gamma$ is primitive but not 2-transitive. For every finite subset $X \subseteq T$, the pointwise stabilizer of $X$ in $\operatorname{Aut}^{0}(T)$ is nontrivial; hence using density again, the same holds for $\Gamma$. The corollary holds.

Now we show that the trichotomy theorem fails for the product of two trees.

Proposition 17. Let $T$ and $U$ be regular trees, both with degree at least 3 . Let $A=\operatorname{Aut}(T) \times \operatorname{Aut}(U)$. Then for every $n \geq 2$ there is a subset $X \subseteq A^{n}$ of infinite measure such that for all $\mathbf{a} \in X$, the closure of the subgroup generated by the elements of $\mathbf{a}$ is not discrete, open or compact.

Proof. Let $\pi_{T}: A \rightarrow \operatorname{Aut}(T)$ and $\pi_{U}: A \rightarrow \operatorname{Aut}(U)$ be the projections to the two coordinates. Let

$$
X=\left\{\mathbf{a} \in A^{n} \mid \pi_{T}(\langle\mathbf{a}\rangle) \text { is dense in } \operatorname{Aut}(T) \text { and } \overline{\pi_{U}(\langle\mathbf{a}\rangle)} \text { is compact }\right\} .
$$

The normalized Haar measure on $A$ equals the product of the normalized Haar measures on $\operatorname{Aut}(T) \times \operatorname{Aut}(U)$, so Theorem 1 implies that $X$ has infinite measure.

Let $\mathbf{a} \in X$, let $\Gamma=\langle\mathbf{a}\rangle$ and let $G$ be the closure of $\Gamma$ in $A$. We claim that $G$ does not satisfy the trichotomy. Indeed, $G$ cannot be compact, since the continuous image $\pi_{T}(G) \supseteq \pi_{T}(\Gamma)$ is dense in $\operatorname{Aut}(T)$ and hence is not compact. Using density again, there exists a sequence $\left(\gamma_{i}\right)$ of distinct elements of $\Gamma$ such that $\left(\pi_{T}\left(\gamma_{i}\right)\right)$ is convergent. Since $\overline{\pi_{U}(\Gamma)}$ is compact, there is a subsequence $\left(\delta_{i}\right)$ of $\left(\gamma_{i}\right)$ such that $\left(\pi_{U}\left(\delta_{i}\right)\right)$ is convergent. But then $\left(\delta_{i}\right)$ is convergent, so $\Gamma$ (and hence $G$ ) is not discrete.

Finally, assume by contradiction that $G$ is open. Then $O=\pi_{U}(G)$ is an open subgroup of $\operatorname{Aut}(U)$, and since $\Gamma$ is dense in $G, \pi_{U}(\Gamma)$ is dense in $O$. This implies that $O$ is topologically finitely generated. The group $O$ is also compact, being an open subgroup of $\overline{\pi_{U}(\Gamma)}$. But then $O$ must stabilize a vertex or a geometric edge. This means that there is an open subgroup of $O$ of index at most 2 that fixes a vertex $t \in T$, which implies that the vertex stabilizer $\operatorname{Aut}(U)_{t}$ has a topologically finitely generated open subgroup. Since $\operatorname{Aut}(U)_{t}$ is compact, we get that $\operatorname{Aut}(U)_{t}$ is itself topologically finitely generated, a contradiction. Hence $G$ is not open. 


\section{ACKNowledgement}

Parts of this paper have been discussed at the Secret Seminar, for which we are openly grateful. This work was supported by the joint BSF grant BSF-2006222. The first author's research was partially supported by NSF grant DMS-0701105. The second author's research was partially supported by ISF grant 888/07.

\section{REFERENCES}

[Ab05] M. Abért, Group laws and free subgroups in topological groups, Bull. London Math. Soc. 37 (2005), no. 4, 525-534. MR2143732 (2006d:20005)

[AV05] M. Abért and B Virág, Dimension and randomness in groups acting on rooted trees, J. Amer. Math. Soc. 18 (2005), no. 1, 157-192. MR2114819 (2005m:20058)

[BaL00] H. Bass and A. Lubotzky, Tree lattices, Progress in Mathematics 176, Birkhauser, 2000. MR $1794898(2001 \mathrm{k}: 20056)$

[BG04] E. Breuillard and T. Gelander, A topological Tits alternative, Annals of Math. 166 (2007), 427-474.

[Bha95] M. Bhattacharjee, The ubiquity of free subgroups in certain inverse limits of groups. J. Algebra 172 (1995), no. 1, 134-146. MR1320624 (96c:20044)

[Dix90] J. D. Dixon, Most finitely generated permutation groups are free, Bull. London Math. Soc. 22 (1990), no. 3, 222-226. MR1041134 (91c:20005)

[FD04] M. Feighn and G. Diao, The Grushko decomposition of a finite graph of finite rank free groups: an algorithm, Preprint, 2004. MR2175158 (2006i:20045)

[Gel07] T. Gelander, On deformations of free subgroups in compact Lie groups, Israel J. Math, 167 (2008), 15-26.

[Gri00] R. I. Grigorchuk, Just infinite branch groups, in New horizons in pro- $p$ groups, 121-179, Progr. Math., 184, Birkhäuser Boston, Boston, MA, 2000. MR.1765119 (2002f:20044)

[FTN91] A. Figà-Talamanca and C. Nebbia, Harmonic analysis and representation theory for groups acting on homogeneous trees, London Mathematical Society Lecture Note Series, vol. 162, Cambridge University Press, Cambridge, 1991. MR.1152801 (93f:22004)

[GG] T. Gelander and Y. Glasner, Countable primitive groups, Geom. Funct. Anal. 17 (2008), no. 5, 1479-1523. MR 2377495 (2008m:20003)

[GSS04] Y. Glasner, J. Souto and P. Storm, Maximal subgroups of lattices in $\mathrm{PSl}_{2} \mathbb{C}$, math.GT/0504441

[KW03] I. Kapovich and R. Weidmann, Nielsen methods and groups acting on hyperbolic spaces, Geom. Dedicata 98 (2003), 95-121. MR1988426 (2004e:20076)

[Lub91] A. Lubotzky, Lattices in rank one Lie groups over local fields, Geom. Funct. Anal. 1 (1991), no. 4, 406-431. MR.1132296 (92k:22019)

[Nie55] J. Nielsen, A basis for subgroups of free groups, Math. Scand. 3 (1955), 31-43. MR0073592(17:455e)

[Pra03] C. E. Praeger, Quotients and inclusions of finite quasiprimitive permutation groups, J. Algebra 269 (2003), no. 1, 329-346. MR2015315 (2004i:20002)

[Ser80] J.-P. Serre, Trees, Springer-Verlag, Berlin, 1980. Translated from the French by John Stillwell. MR 1954121 (2003m:20032)

[Tit70] J. Tits, Sur le groupe des automorphismes d'un arbre, Essays on topology and related topics (Mémoires dédiés à Georges de Rham), Springer, New York, 1970, pp. 188-211. MR 0299534 (45:8582)

[Wei02] R. Weidmann, The Nielsen method for groups acting on trees, Proc. London Math. Soc. (3) 85 (2002), no. 1, 93-118. MR.1901370 (2003c:20029)

Department of Mathematics, University of Chicago, 5734 University Avenue, Chicago, ILLINOIS 60637

Department of Mathematics, Ben Gurion University of the Negev, 84105 Beer Sheva, ISRAEL 